\title{
State-Dependent and Timing-Dependent Bidirectional Associative Plasticity in the Human SMA-M1 Network
}

\author{
Noritoshi Arai, ${ }^{1,2 \star}$ Florian Müller-Dahlhaus, ${ }^{1 \star}$ Takenobu Murakami, ${ }^{1}$ Barbara Bliem, ${ }^{1}$ Ming-Kuei Lu, $, 13,4$ \\ Yoshikazu Ugawa, ${ }^{5}$ and Ulf Ziemann ${ }^{1}$ \\ ${ }^{1}$ Department of Neurology, Goethe-University Frankfurt, 60528 Frankfurt am Main, Germany, ${ }^{2}$ Department of Neurology, Graduate School of Medicine, \\ University of Tokyo, Tokyo, 113-8654, Japan, ${ }^{3}$ Neuroscience Laboratory, Department of Neurology, China Medical University Hospital, Taichung 40447, \\ Taiwan, ${ }^{4}$ Institute of Medical Science and School of Medicine, China Medical University, Taichung 40402, Taiwan, and ${ }^{5}$ Department of Neurology, School of \\ Medicine, Fukushima Medical University, 960-1295 Fukushima, Japan
}

The supplementary motor area (SMA-proper) plays a key role in the preparation and execution of voluntary movements. Anatomically, SMA-proper is densely reciprocally connected to primary motor cortex (M1), but neuronal coordination within the SMA-M1 network and its modification by external perturbation are not well understood. Here we modulated the SMA-M1 network using MR-navigated multicoil associative transcranial magnetic stimulation in healthy subjects. Changes in corticospinal excitability were assessed by recording motor evoked potential (MEP) amplitude bilaterally in a hand muscle. We found timing-dependent bidirectional Hebbian-like MEP changes during and for at least $30 \mathrm{~min}$ after paired associative SMA-M1 stimulation. MEP amplitude increased if SMA stimulation preceded M1 stimulation by $6 \mathrm{~ms}$, but decreased if SMA stimulation lagged M1 stimulation by $15 \mathrm{~ms}$. This associative plasticity in the SMA-M1 network was highly topographically specific because paired associative stimulation of pre-SMA and M1 did not result in any significant MEP change. Furthermore, associative plasticity in the SMA-M1 network was strongly state-dependent because it required priming by near-simultaneous M1 stimulation to occur. We conclude that timing-dependent bidirectional associative plasticity is demonstrated for the first time at the systems level of a human corticocortical neuronal network. The properties of this form of plasticity are fully compatible with spike-timing-dependent plasticity as defined at the cellular level. The necessity of priming may reflect the strong interhemispheric connectivity of the SMA-M1 network. Findings are relevant for better understanding reorganization and potentially therapeutic modification of neuronal coordination in the SMA-M1 network after cerebral lesions such as stroke.

\section{Introduction}

The supplementary motor area (SMA) is crucial for preparation and execution of voluntary movements and may play an important role in linking cognition to action (Nachev et al., 2008). Based on microstructure, white-matter connectivity profiles, and function, two distinct SMA areas can be distinguished: the caudal SMA-proper and the rostrally adjacent pre-SMA (Picard and Strick, 1996; Geyer et al., 2000; Johansen-Berg et al., 2004). In monkeys, SMA-proper has tight reciprocal connections with bilateral primary motor cortex (M1) (Luppino et al., 1993; Geyer et al., 2000; Dum and Strick, 2005) and is strongly interconnected via transcallosal fibers (Rouiller et al., 1994; Liu et al., 2002), whereas pre-SMA is not directly connected with M1, neither ipsilaterally nor transcallosally (Luppino et al., 1993; Geyer et al., 2000; Liu et al., 2002). In humans, neuroimaging and physiolog-

\footnotetext{
Received May 6, 2011; revised Aug. 11, 2011; accepted Sept. 6, 2011.

Author contributions: U.Z. designed research; N.A., T.M., B.B., and M.-K.L. performed research; N.A., F.M.-D., T.M., and U.Z. analyzed data; N.A., F.M.-D., Y.U., and U.Z. wrote the paper.

This study was supported by a grant from Sankyo Foundation of Life Science in Japan (N.A.).

*N.A. and F.M.-D. contributed equally to this work.

The authors declare no competing financial interests.

Correspondence should be addressed to Prof. Ulf Ziemann, Department of Neurology, Goethe-University, Schleusenweg 2-16, 60528 Frankfurt am Main, Germany. E-mail: u.ziemann@em.uni-frankfurt.de.

DOI:10.1523/JNEUROSCI.2271-11.2011

Copyright $\odot 2011$ the authors $\quad 0270-6474 / 11 / 3115376-08 \$ 15.00 / 0$
}

ical data provide evidence for a similar reciprocal connectivity pattern between SMA-proper and M1 (Fox et al., 1997; Geyer et al., 2000; Bestmann et al., 2003; Matsumoto et al., 2007; Kim et al., 2010). In contrast, less is known about the functional and effective connectivity in the SMA-M1 network and its dynamic modifiability by external perturbation.

Conventional paired associative stimulation (PAS) consists of repetitive pairing of an electrical peripheral nerve stimulus and focal transcranial magnetic stimulation (TMS) of the contralateral M1 and induces long-term bidirectional changes in M1 excitability, depending on the interval between the two associative stimuli (for review, see Müller-Dahlhaus et al., 2010). The critical dependence of the direction of PAS effects on the exact timing between the two associative stimuli is reminiscent of spike timing-dependent plasticity (STDP), where long-term potentiation occurs if action potentials consistently follow EPSPs, whereas long-term depression occurs if the order of these events is reversed (Markram et al., 1997; Bi and Poo, 2001; Dan and Poo, 2004; Caporale and Dan, 2008). Recent PAS protocols introduced repeated paired-coil focal TMS to modify corticocortical networks. Associative stimulation of homologous areas of left and right $\mathrm{M} 1$ resulted in an interstimulus interval-specific long-term MEP increase in the conditioned M1 but a MEP decrease could not be demonstrated (Koganemaru et al., 2009; Rizzo et al., 2009). Therefore, it is presently uncertain to which extent these findings are truly STDP-like. 
A

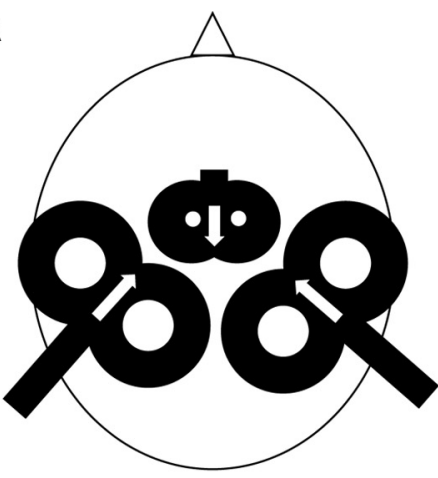

B

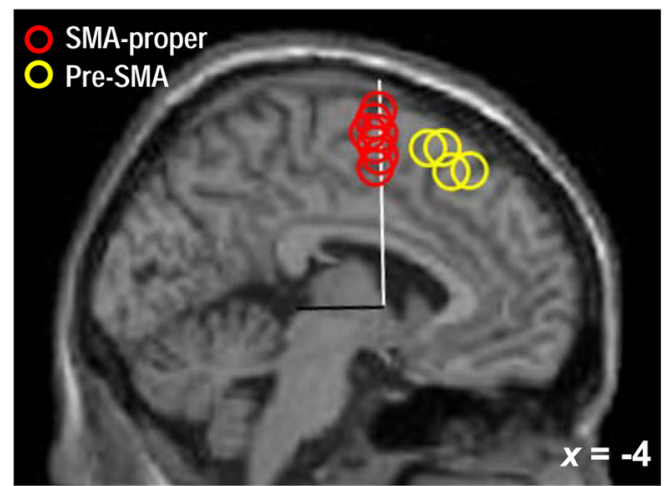

C

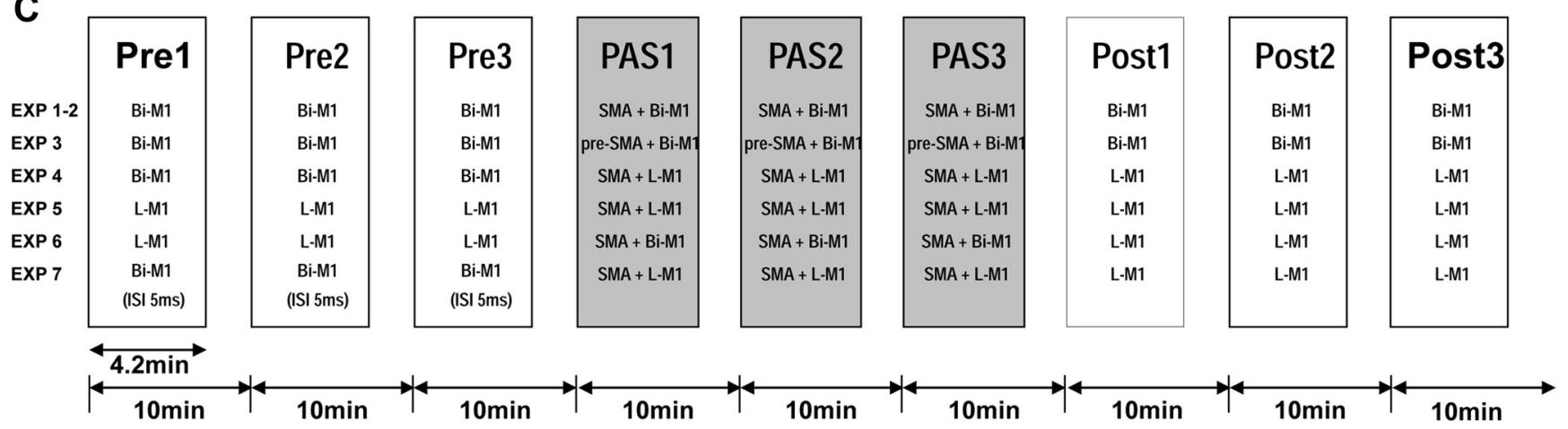

Figure 1. Methodology and experimental design. $A$, Multicoil focal TMS. Near-simultaneous bilateral M1 stimulation (ISI $=0.8 \mathrm{~ms}$; left M1 always before right M1, stimulus intensities adjusted to elicit MEP of $1 \mathrm{mV}$ peak-to-peak amplitude in the contralateral FDI) was delivered through $50 \mathrm{~mm}$ diameter figure-of-eight coils. During paired associative stimulation (PAS1-3, see $\mathbf{C}$ ), SMA-proper was stimulated time-locked to bilateral M1 stimulation using a small $25 \mathrm{~mm}$ figure-of-eight coil (stimulus intensity set to $140 \%$ of AMT as determined over left M1). Directions of induced currents in the brain were as indicated by the white arrows. For further details, see Materials and Methods. B, Sagittal view at $x=-4$ in Talairach stereotaxic space (Talairach and Tournoux, 1988) of the mean anatomical image indicating SMA-proper stimulation sites (center of stimulating coil, red circles) and pre-SMA stimulation sites (yellow circles) during PAS1-3 in those subjects ( $n=$ 7 for SMA-proper, $n=4$ for pre-SMA) in whom MR-navigation was used to verify stimulation topography. (ircles are artificially stacked along the $z$-axis to provide visibility of all individual stimulation sites. The vertical plane through the anterior commissure (white line, $y=0$ ) and the perpendicular anterior-posterior commissure plane (black line) are indicated. Mean coordinates for the SMA-proper stimulation sites were $y=-1.3 \pm 0.6$ (Talairach and Tournoux, 1988) and $3.3 \pm 0.2 \mathrm{~cm}$ anterior to $C_{Z^{\prime}}$ mean coordinates for the pre-SMA stimulation sites were $y=23.7 \pm 3.3$ (Talairach and Tournoux, 1988) and $6.2 \pm 3.2 \mathrm{~cm}$ anterior to $\mathrm{C}_{2}$. C, General time line of Experiment 1-7, always consisting of nine blocks of 50 trials each (Pre1-3, PAS1-3, Post1-3). Pre1-3 measure M1 excitability at baseline, PAS1-3 apply paired associative stimulation of SMA and M1, and Post1-3 assess any lasting effects on M1 excitability after PAS1-3. Variations of the stimulation procedures in Experiments 1-7 are detailed in Materials and Methods. EXP, Experiment; Bi-M1, bilateral M1 stimulation; L-M1, left M1 stimulation; SMA, SMA-proper.

Here we introduce a novel multicoil focal associative stimulation protocol to target the SMA-proper and bilateral M1 network. Due to the particular role of this network in bimanual coordination and its strong interhemispheric connectivity we deliberately set out to apply associative stimulation of SMA-proper paired with near-simultaneous bilateral M1 stimulation. We demonstrate state-dependent and timing-dependent, topographically specific bidirectional Hebbian-like associative plasticity in the SMA-M1 network. Recent studies showed a significant role of effective coupling of neuronal activity in the SMA-M1 network for motor outcome in patients after stroke (Grefkes et al., 2008, 2010; Wang et al., 2011). In this context, the present study may signify therapeutic potential to support purposeful modification of coupling in the SMA-M1 network in neurological disorders in which dysfunction of this network contributes to the clinical impairment.

\section{Materials and Methods}

\section{Subjects}

Twenty-nine healthy subjects participated in the study (mean age \pm SEM, $28.2 \pm 1.5$ years; age range, $19-43$ years; 18 female). Varying subgroups of subjects participated in the different experiments (Experiments $1-7$, see below). Some subjects participated in more than one experiment. In these cases, the interval between successive experimental sessions was at least 1 week to avoid carryover effects. None of the subjects had a history of neurological disease or was on CNS active drugs at the time of the experiments. All subjects were right-handed according to the Edinburgh Handedness Inventory (Oldfield, 1971) except for two who were left-handed. Written informed consent was obtained before participation. The experiments conformed to the Declaration of Helsinki and were approved by the ethics committee of the medical faculty of Goethe University, Frankfurt.

\section{EMG recordings}

Surface electromyography (EMG) was recorded from resting right and left first dorsal interosseus (FDI) muscles using pairs of electrodes in a belly-tendon montage. The EMG raw signal was amplified, rectified and filtered at a bandpass of $30-200 \mathrm{~Hz}$ (Counterpoint Mk2, Dantec). EMG signals were digitized at a rate of $1 \mathrm{kHz}$ and stored for offline analysis. During all recordings, subjects were requested to relax but stay awake. Complete voluntary muscle relaxation was monitored audio-visually by high-gain EMG (50 $\mu \mathrm{V} /$ division).

\section{Transcranial magnetic stimulation}

Focal TMS was applied over the hand area of left and right M1 through figure-of-eight coils (outer diameter of each wing, $50 \mathrm{~mm}$ ) using two Magstim 200 magnetic stimulators (Magstim Company) with monophasic current waveforms. Coils were held tangential to the scalp and rotated away from the midline by $45^{\circ}$ so that the induced currents in M1 were directed from lateral-posterior to medial-anterior (Fig. 1A). This is the optimal current direction to excite corticospinal neurons transsynaptically via excitatory interneurons (Sakai et al., 1997; Di Lazzaro et al., 2008). The optimal coil position for eliciting MEP in the FDI muscles was determined as the site, where stimulation at a slightly suprathreshold stimulus intensity produced consistently the largest MEP amplitudes. 
Table 1. Means ( \pm SEM) of test MEP amplitudes (in $\mathrm{mV}$ ) at baseline (time point Pre1) in Experiments 1-7

\begin{tabular}{lll}
\hline Experiment & Left M1 & Right M1 \\
\hline $\begin{array}{lll}\text { Experiment 1 } \\
|S|-6 \mathrm{~ms}\end{array}$ & $1.03 \pm 0.10$ & \\
$\mid \mathrm{SI}-3.2 \mathrm{~ms}$ & $1.12 \pm 0.15$ & $0.85 \pm 0.09$ \\
$|\mathrm{~S}|+10 \mathrm{~ms}$ & $1.01 \pm 0.11$ & $1.08 \pm 0.15$ \\
$|\mathrm{~S}|+15 \mathrm{~ms}$ & $1.34 \pm 0.15$ & $1.30 \pm 0.16$ \\
Experiment 2 & $1.18 \pm 0.10$ & $1.46 \pm 0.16$ \\
Experiment 3 & $1.17 \pm 0.13$ & $1.18 \pm 0.13$ \\
Experiment 4 & $0.97 \pm 0.06$ & $1.19 \pm 0.14$ \\
Experiment 5 & $0.93 \pm 0.13$ & n.d. \\
Experiment 6 & $1.03 \pm 0.07$ & n.d. \\
Experiment 7 & $1.30 \pm 0.30$ & n.d. \\
\hline
\end{tabular}

n.d., Not done.

The two stimulation sites were marked on the scalp with a felt-tip pen to ensure consistent placement of the coils throughout the experiment. For near-simultaneous bilateral M1 stimulation, TMS pulses to the left and right $\mathrm{M} 1$ were separated by $0.8 \mathrm{~ms}$ to avoid physical interference between the two stimuli (Cincotta et al., 2005). Left M1 stimulation always preceded right M1 stimulation. Resting motor threshold for both FDI was determined to the nearest $1 \%$ of maximum stimulator output (MSO) as the lowest stimulus intensity that elicited small MEP $(>50 \mu \mathrm{V}$ peak-topeak amplitude) in at least five of 10 consecutive trials. Intensity of left and right M1 stimulation was adjusted to evoke MEP of $\sim 1 \mathrm{mV}$ peakto-peak amplitude at baseline (time point Pre1, see below). Table 1 shows that this target MEP amplitude was relatively well matched in all experiments (Experiment 1-7). Statistical analysis did not reveal significant differences in baseline MEP amplitude between experiments. This is an important nil finding because it excludes that differences in MEP amplitude at baseline accounted for the differences in associative plasticity in the SMA-M1 network observed between experiments (see Results).

The SMA was stimulated using a small figure-of-eight coil (outer diameter of each wing, $25 \mathrm{~mm}$, Magstim Company) connected to a third Magstim 200 magnetic stimulator. After placement of the left and right M1 coils (see above), the SMA coil was centered on the midline as posterior as possible to fit between the two M1 coils (Fig. $1 A$ ). The distance between vertex (electrode position $\mathrm{C}_{\mathrm{z}}$ of the International 10-20 EEG system) and the intersection of the two wings of the small coil (hot spot of SMA stimulation) was measured at the beginning of every experimental session and in every subject. Only subjects with distances $\leq 4.0 \mathrm{~cm}$ were included because larger distances likely target the pre-SMA rather than SMA-proper (Picard and Strick, 1996; Fink et al., 1997; Matsunaga et al., 2005; Mars et al., 2009). The mean distance \pm SEM (across all experiments except Experiment 3 which targeted pre-SMA) was $3.64 \pm 0.05$ $\mathrm{cm}$. In seven subjects we verified correct targeting of SMA-proper by visualizing coil placement on individual brain anatomy using a frameless MR-guided TMS navigation system (Localite TMS Navigator, Localite $\mathrm{GmbH})$. In all seven subjects, the center of the coil junction was located over SMA-proper which was separated from the anteriorly adjacent preSMA by a vertical line from the anterior commissure (VCA line, Fig. $1 B$ ) perpendicular to the anterior-posterior commissure line in the sagittal plane (Picard and Strick, 1996; Zilles et al., 1996; Vorobiev et al., 1998). The induced current within SMA-proper flowed in the antero-toposterior direction (Fig. 1A) (Civardi et al., 2001). Stimulus intensity was related to active motor threshold (AMT) as determined by the small SMA-coil over left M1. AMT was determined during a slight isometric contraction of the right FDI ( $\sim 10 \%$ of maximum voluntary contraction, monitored by audio-visual feedback of the EMG signal) and measured to the nearest $1 \%$ of MSO as the lowest stimulus intensity which produced a mean MEP of $>100 \mu \mathrm{V}$ from the curve average of five consecutive single-trial sweeps (Ziemann et al., 1996). SMA stimulation intensity was set to $140 \%$ of AMT except for Experiment 2 ( $90 \%$ of AMT).

\section{Experimental design}

Subjects were seated on a comfortable reclining chair with both arms relaxed. All experiments consisted of nine separate blocks of 50 trials each
(Fig. 1C). In blocks 1-3 (Pre1-3), unilateral or near-simultaneous bilateral M1 stimulation was delivered without SMA stimulation. In blocks 4-6 (PAS1-3), multicoil associative TMS of SMA and unilateral or nearsimultaneous bilateral M1 stimulation was applied. In blocks 7-9 (Post1-3), unilateral or near simultaneous bilateral M1 stimulation without SMA stimulation was repeated to probe changes in MEP amplitude induced by PAS1-3. In each block, the intertrial interval was set to $5 \mathrm{~s} \pm 25 \%$ variation to reduce anticipation of the next trial. The mean duration of each block of trials was $4.2 \mathrm{~min}$. The interval between the onsets of consecutive blocks of trials was 10 min to allow for cooling of the stimulation coils. In total, seven different experiments (Experiments 1-7) were conducted. Numbers of subjects in each experiment and mean \pm SEM distances $(d)$ from $\mathrm{C}_{\mathrm{z}}$ to SMA-coil are indicated in brackets.

Experiment 1. During PAS1-3, TMS of SMA-proper was given in separate experiments before [interstimulus intervals (ISIs) of $-6 \mathrm{~ms}(\mathrm{~N}=7$, $d=3.57 \pm 0.20 \mathrm{~cm})$ or $-3.2 \mathrm{~ms}(N=7, d=3.86 \pm 0.09 \mathrm{~cm})]$ or after [ISI $+10 \mathrm{~ms}(N=8, d=3.67 \pm 0.15 \mathrm{~cm})$ or $+15 \mathrm{~ms}(N=7, d=3.74 \pm$ $0.17 \mathrm{~cm})]$ near-simultaneous $(\Delta t=0.8 \mathrm{~ms})$ bilateral M1 stimulation. For those subjects who participated in more than one session in Experiment 1, the order of sessions was pseudorandomized to exclude a possible order effect. The ISIs in this experiment and in Experiments 2-3 and 6 refer to the delay between SMA and the middle time between left and right M1 stimulation, and negative ISIs indicate that SMA stimulation preceded M1 stimulation (for instance, the ISI of -6 ms indicates that SMA stimulation occurred $5.6 \mathrm{~ms}$ before left and $6.4 \mathrm{~ms}$ before right M1 stimulation). At the ISI of $-6 \mathrm{~ms}$ excitatory synaptic input from SMA stimulation is thought to arrive in M1 coincidently with or shortly before generation of action potentials in corticospinal neurons by $\mathrm{M} 1$ stimulation, while the order of these events in M1 is reversed at the ISI of $+15 \mathrm{~ms}$. This rationale is based on short-latency, likely mono-synaptic excitatory input from SMA-proper to M1 corticospinal neurons (peaking at $2 \mathrm{~ms}$ and 4-6 ms) in monkey intracortical microstimulation experiments (Tokuno and Nambu, 2000) and paired-coil experiments from our group, which showed MEP facilitation if stimulation of SMA-proper preceded a TMS test pulse over M1 by $6 \mathrm{~ms}$ (N. Arai, M.-K. Lu, Y. Ugawa, and U. Ziemann, unpublished data). The ISIs of $-3.2 \mathrm{~ms}$ and $+10 \mathrm{~ms}$ were selected because conventional PAS experiments pairing electrical peripheral nerve stimulation with TMS of the contralateral M1 or primary somatosensory cortex had demonstrated a steep transition from a maximum long-term facilitation to maximum depression within a time window of 15-20 ms (Wolters et al., 2003, 2005). If this time window also applied to PAS-induced plasticity in the SMA-M1 network, then these intervals, which are intermediate to $-6 \mathrm{~ms}$ and $+15 \mathrm{~ms}$, should be less effective in inducing long-term MEP change.

As we observed timing-dependent bidirectional associative plasticity in Experiment 1 (see Results), all other experiments (Experiments 2-7) were designed to examine in detail the physiological characteristics of this associative plasticity in the SMA-M1 network.

Experiment $2(N=6, d=3.69 \pm 0.17 \mathrm{~cm})$. This experiment was performed to test the effect of SMA-proper stimulation at a lower intensity of $90 \%$ of AMT. The ISI between SMA and near-simultaneous bilateral M1 stimulation was set at $-6 \mathrm{~ms}$. Under these conditions, a paired-coil experiment showed no effect of conditioning SMA stimulation on test MEP amplitude (N. Arai, M.-K. Lu, Y. Ugawa, and U. Ziemann, unpublished data). Therefore, we expected that associative plasticity (long-term MEP increase) as observed with $140 \%$ of AMT stimulation of SMAproper in Experiment 1 should no longer occur.

Experiment $3(N=9, d=6.27 \pm 0.27 \mathrm{~cm})$. This experiment tested the topographical specificity of the findings in Experiment 1 . The stimulation site over the midline was moved either $3 \mathrm{~cm}$ anterior from the individually determined site for SMA-proper stimulation as described above $(n=7)$ or deliberately targeted pre-SMA at $y=18$ in Talairach space (Talairach and Tournoux, 1988) using MR-navigation $(n=2)$. MR-navigation was performed in altogether 4 subjects. All stimulation sites were located anterior to the VCA line $(y=23.7 \pm 3.2$ in Talairach space, Fig. $1 B$ ) confirming correct placement of the stimulating coil over the pre-SMA (Picard and Strick, 1996). Otherwise, Experiment 3 was identical to Experiment 1 (ISI between pre-SMA and near-simultaneous 
M1 stimulation: $-6 \mathrm{~ms}$; intensity of pre-SMA stimulation: $140 \%$ of AMT).

Experiment $4(N=7, d=3.56 \pm 0.22 \mathrm{~cm})$. This experiment explored the necessity of pairing bilateral M1 stimulation with SMA-proper stimulation to obtain the long-term MEP increase observed in Experiment 1. Hence, during PAS1-3, SMA-proper stimulation was paired with unilateral left M1 stimulation rather than near-simultaneous bilateral M1 stimulation. The ISI between SMA-proper and left M1 stimulation was $-5.6 \mathrm{~ms}$ (i.e., the same as in Experiment 1). Pre1-3 constituted of nearsimultaneous M1 stimulation, while during Post1-3 only unilateral left M1 stimulation was conducted.

Experiment $5(N=7, d=3.55 \pm 0.14 \mathrm{~cm})$. This experiment was designed according to the long-term MEP increase obtained in Experiments 1 and 4 and tested the necessity of near-simultaneous bilateral M1 stimulation during Pre1-3 to induce this form of associative plasticity during PAS1-3. Thus, Pre1-3 (and Post1-3) consisted of unilateral left M1 rather than near-simultaneous bilateral M1 stimulation, and PAS1-3 consisted of SMA-proper stimulation paired with unilateral left M1 stimulation at the ISI of $-5.6 \mathrm{~ms}$.

Experiment $6(N=7, d=3.60 \pm 0.18 \mathrm{~cm})$. This experiment also addressed the necessity of near-simultaneous bilateral M1 stimulation during Pre1-3 to induce long-term MEP increase during PAS1-3. The experiment was identical to Experiment 5 with the exception that PAS1-3 consisted of pairing SMA-proper stimulation with nearsimultaneous bilateral M1 stimulation.

Experiment $7(N=7, d=3.60 \pm 0.18 \mathrm{~cm})$. This experiment examined to which extent the synchronicity of bilateral M1 during Pre1-3 was important to induce long-term MEP increase during PAS1-3. Toward this end, Pre1-3 consisted of bilateral M1 stimulation at an ISI of $5 \mathrm{~ms}$ (i.e., nonsimultaneous stimulation of left M1 before right M1). PAS1-3 consisted of SMA-proper stimulation paired with unilateral left M1 stimulation at the ISI of $-5.6 \mathrm{~ms}$. During Post1-3, unilateral left M1 stimulation was applied.

\section{Data analysis and statistics}

For each of the nine blocks of trials (Pre1-3, PAS1-3, and Post1-3) the single-trial measured peak-to-peak MEP amplitudes were averaged to obtain mean MEP amplitudes for each time point. MEP amplitudes at time points Pre2-3, PAS1-3, and Post1-3 were normalized to the mean MEP amplitude at time point Pre1. The Shapiro-Wilk test was performed to test data for normal distribution. In all cases the null hypothesis had to be rejected, thus the normalized MEP amplitudes were logarithmized to homogenize the variances before averaging. In Experiment 1 , a repeated-measures ANOVA (rmANOVA) was applied to test the within-subject effects of Time (here and in all following experiments, nine levels: Pre1-3, PAS1-3, Post1-3) and Side (two levels: left M1, right $\mathrm{M1}$ ) and the between-subject effect of ISI (four levels: $-6 \mathrm{~ms},-3.2 \mathrm{~ms}$, $+10 \mathrm{~ms},+15 \mathrm{~ms}$ ). In Experiment 2, rmANOVA tested the withinsubject effects of Time and Side and the between-subject effect of stimulation Intensity (two levels: 90\% and 140\% of AMT). In Experiment 3, rmANOVA tested the within-subject effects of Time and Side and the between-subject effect of stimulation Site (two levels: pre-SMA, SMAproper). In Experiment 4, rmANOVA tested the within-subject effect of Time and the between-subject effect of Experiment (two levels: Experiment 4, Experiment 1). rmANOVAs of Experiments 2-4 used the data from Experiment 1 (ISI of $-6 \mathrm{~ms}$ ) for comparison because they differed always in only one experimental detail (stimulation intensity, stimulation site, or PAS of SMA with unilateral rather than bilateral M1 stimulation, cf. Fig. 1C). In Experiments 5-7, rmANOVAs tested the within-subject effect of Time and the between-subject effect of Experiment (always two levels, Experiment 5-7 vs Experiment 4). Experiment 4 was chosen for comparison because Experiments 5-7 were specific modifications of Experiment 4 (cf. Fig. 1C).

In case of a significant main effect, post hoc testing was performed using Fisher's PLSD to examine differences between the single levels of this effect. All statistical analyses were conducted using StatView for Windows 5.0.1. software (SAS Institute Inc.). In all tests statistical significance was assumed if $p<0.05$. If not stated otherwise, data are expressed as mean \pm 1 SEM.

\section{Results}

Experiment 1: timing-dependent bidirectional PAS-induced plasticity in the SMA-M1 network

There was a significant effect of ISI $\left(F_{(3,24)}=20.94, p<0.0001\right)$ and the interaction of ISI with Time $\left(F_{(24,192)}=2.15, p=0.002\right)$ while the effect of Side and its interactions with ISI, and Time were not significant (all $p>0.15$ ). Post hoc comparisons between ISIs showed that the ISI of $-6 \mathrm{~ms}$ was different from all other ISIs (all $p<0.0001$ ), the ISI of -3.2 ms was different from the ISI of $+15 \mathrm{~ms}(p=0.0055)$, and the ISI of $+10 \mathrm{~ms}$ was different from the ISI of $+15 \mathrm{~ms}(p=0.0086)$, while the ISI of $-3.2 \mathrm{~ms}$ was not different from the ISI of $+10 \mathrm{~ms}(p=0.77)$ (Fig. 2). Post hoc one-sample $t$ tests revealed significant MEP increases throughout time points PAS1-Post 3 for the ISI of $-6 \mathrm{~ms}$ and significant MEP decreases at time points PAS1, Post 2 and Post 3 for the ISI of +15 $\mathrm{ms}(p<0.05)$, while no significant MEP change occurred with the ISI of $-3.2 \mathrm{~ms}$ and $+10 \mathrm{~ms}$ (Fig. 2). The findings demonstrate lasting timing-dependent bidirectional PAS-induced excitability change in M1 induced by PAS of the SMA-M1 network.

\section{Experiment 2: effect of SMA stimulation intensity on PAS-induced plasticity in the SMA-M1 network}

There was a significant effect of Intensity $\left(F_{(1,11)}=24.37, p=\right.$ $0.0004)$ and its interaction with Time $\left(F_{(8,88)}=3.48, p=0.0016\right)$ while the effect of Side and its interactions with Intensity and Time were not significant (all $p>0.4$ ) (Fig. 3A). Post hoc unpaired $t$ tests showed significant differences between stimulation intensity $140 \%$ versus $90 \%$ of AMT throughout time points PAS1-Post3 $(p<0.005)$. Findings indicate that the lasting M1 excitability increase induced by PAS of the SMA-M1 network requires SMA stimulation of sufficient strength.

\section{Experiment 3: topographic specificity of PAS-induced plasticity in the SMA-M1 network}

There was a significant effect of stimulation Site $\left(F_{(1,14)}=28.33\right.$, $p=0.0001)$ and its interaction with Time $\left(F_{(8,112)}=3.41, p=\right.$ $0.0015)$ while the effect of Side and its interactions with Site and Time were not significant (all $p>0.6$ ) (Fig. 3B). Post hoc unpaired $t$ tests showed significant differences between stimulation of SMA-proper vs pre-SMA throughout time points PAS1-Post3 $(p<0.005)$. Findings indicate that the lasting M1 excitability increase induced by PAS of the SMA-M1 network is topographically specific as it occurs with stimulation of SMA-proper but not pre-SMA.

Experiments 4-7: priming by near-synchronous bilateral M1 stimulation is necessary for induction of associative plasticity in the SMA-M1 network

Near-simultaneous M1 stimulation during Pre1-3 and pairing SMA-proper with unilateral left M1 stimulation at the ISI of -6 ms during PAS1-3 (Experiment 4) resulted in a significant effect of Time $\left(F_{(6,48)}=7.35, p<0.0001\right)$, which was explained in post hoc one-sample $t$ tests by significant MEP increases throughout time points PAS1-Post3 (all $p<0.05$ ) (Fig. $4 A$ ). Direct comparison with near-simultaneous M1 stimulation during Pre1-3 and pairing SMA-proper with bilateral M1 stimulation at the ISI of -6 ms during PAS1-3 (Experiment 1) showed no significant effect of Experiment $\left(F_{(1,12)}=0.36, p=0.56\right)$ or the interaction of Experiment with Time $\left(F_{(8,96)}=1.11, p=0.36\right)$ (Fig. $\left.4 A\right)$. Findings indicate that pairing of SMA-proper stimulation with unilateral M1 stimulation is sufficient for associative plasticity in the SMA-M1 network to occur. 


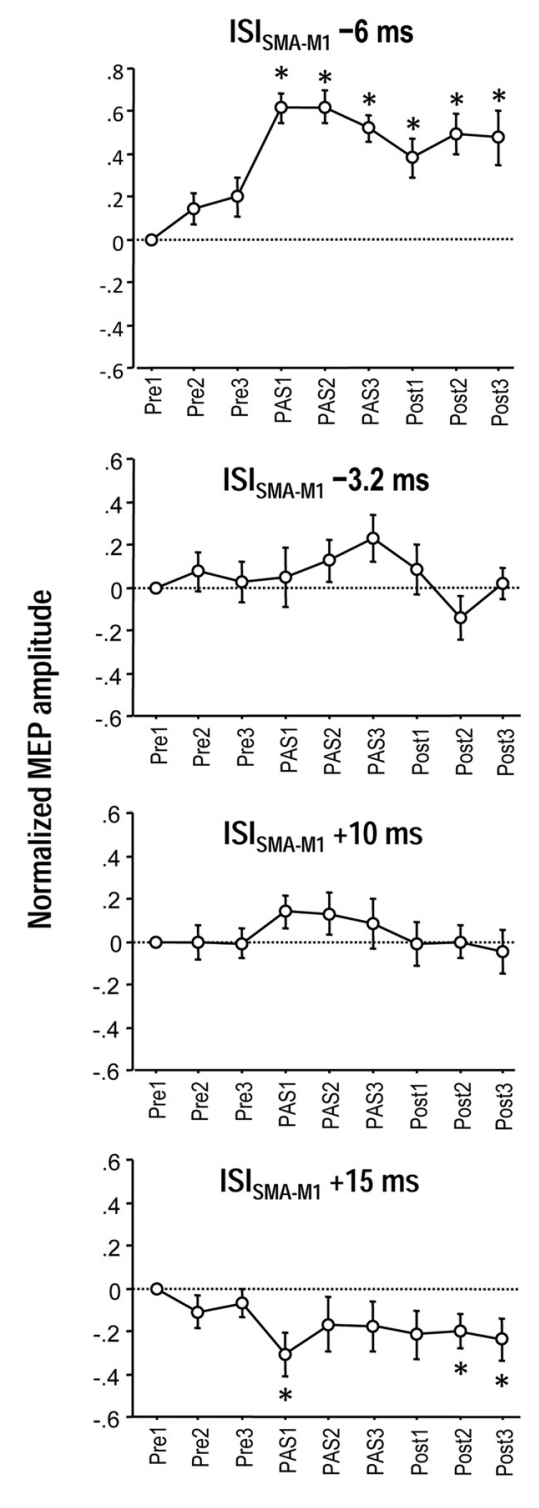

Time points of MEP measurements

Figure 2. Timing-dependent associative plasticity between SMA and bilateral M1 (Experiments 1). MEP changes before (Pre1-3), during (PAS1-3) and after (Post1-3) associative stimulation of SMA paired with near-simultaneous stimulation of bilateral M1. MEP amplitudes increased significantly during and for at least $30 \mathrm{~min}$ after PAS1-3 at the ISI of $-6 \mathrm{~ms}$ (SMA before bilateral M1 stimulation, top). In contrast, MEP amplitudes decreased for at least $30 \mathrm{~min}$ after PAS1-3 at the ISI of +15 ms (SMA after bilateral M1 stimulation, bottom panel). No significant MEP changes occurred with the ISI of $-3.2 \mathrm{~ms}$ (second panel from top) and the ISI of $+10 \mathrm{~ms}$ (second panel from bottom). The MEP changes at the ISI of $-6 \mathrm{~ms}$ were significantly different from all other ISIs, those at the ISI of $-3.2 \mathrm{~ms}$ were different from the $I S I$ of $+15 \mathrm{~ms}$, and those of the ISI of $+10 \mathrm{~ms}$ were different from the $I S I$ of $+15 \mathrm{~ms}$ (all $p<0.009$ ). All MEP data were pooled from the left and right M1 because the rmANOVAs did not show an effect of Side (left vs right M1) or its interactions with any other main effect. Asterisks denote significant MEP changes compared with Pre 1 ( $p<0.05$, post hoct tests). MEP data in this and all following figures are logarithmized and normalized to Pre1, and shown as means \pm SEM.

The data from Experiment 4 served as reference for Experiments 5-7. Compared with Experiment 4, near-simultaneous bilateral M1 stimulation during Pre1-3 was modified to unilateral left M1 stimulation in Experiment 5 (Fig. 1C). There were significant effects of Experiment $\left(F_{(1,12)}=5.28, p=0.040\right)$ and the interaction of Experiment with Time $\left(F_{(8.96)}=2.04, p=0.049\right)$ (Fig. 4B). Post hoc unpaired $t$ tests revealed significant differ-
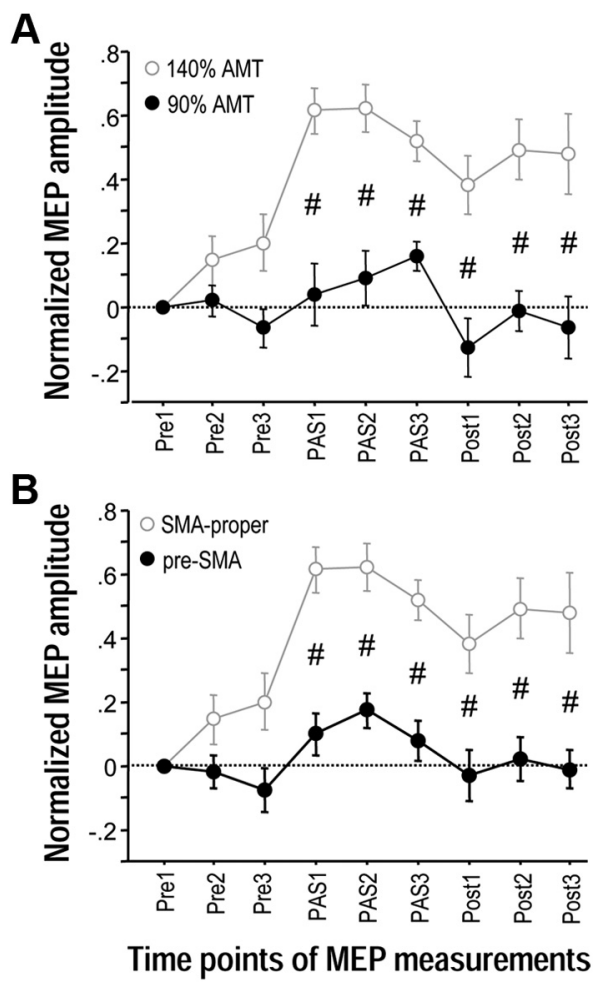

Figure 3. Effects of SMA stimulation intensity and topographic specificity of associative plasticity between SMA and bilateral M1 (Experiments 2 and 3). A, MEP changes before (Pre13), during (PAS1-3) and after (Post1-3) associative stimulation of SMA paired with nearsimultaneous stimulation of bilateral M1. The intensity of SMA stimulation was reduced to $90 \%$ of AMT (black circles). Data are compared with $140 \%$ of AMT (gray circles, data from Experiment 1). The ISI between SMA and bilateral M1 stimulation was $-6 \mathrm{~ms}$. $\boldsymbol{B}$, MEP changes before (Pre1-3), during (PAS1-3) and after (Post1-3) associative stimulation of SMA paired with near-simultaneous stimulation of bilateral $M 1$ at a stimulation site $3 \mathrm{~cm}$ anterior to the individually determined SMA-proper (i.e., corresponding to pre-SMA, black circles). Stimulation intensity was $140 \%$ of AMT, and ISI between SMA and bilateral M1 stimulation was -6 ms. Data are compared with SMA-proper stimulation (gray circles, data from Experiment 1). MEP data were pooled from the left and right $M 1$ because the rmANOVAs did not show an effect of Side (left vs right M1) or its interactions with any other main effect. Significant difference of MEP changes at a single time point between the experimental conditions, ${ }^{\#} p<0.005$, unpaired $t$ tests. Note that lasting MEP increase critically depends on efficient stimulation of SMA-proper and does not occur with low stimulation intensity or stimulation of pre-SMA.

ences between Experiment 4 and Experiment 5 at time points Post 2 and Post $3(p<0.05$, Fig. $4 B)$. Experiment 6 used unilateral left M1 stimulation during Pre1-3 and pairing of SMA with bilateral M1 stimulation during PAS1-3 (Fig. 1C). There were significant effects of Experiment $\left(F_{(1,12)}=8.02, p=\right.$ $0.015)$ and the interaction of Experiment with Time $\left(F_{(8.96)}=\right.$ $3.14, p=0.0034)$ (Fig. 4C). Post hoc unpaired $t$ tests revealed significant differences between Experiment 4 and Experiment 6 at time points PAS2, Post2 and Post3 ( $p<0.05$, Fig. $4 C)$.

Finally, Experiment 7 modified Pre1-3 to bilateral but nonsimultaneous M1 stimulation but otherwise was identical to Experiment 4 (Fig. 1C). There were significant effects of Experiment $\left(F_{(1,12)}=13.31, p=0.0033\right)$ and the interaction of Experiment with Time $\left(F_{(8.96)}=6.88, p<0.0001\right)$ (Fig. $\left.4 D\right)$. Post hoc unpaired $t$ tests revealed significant differences between Experiment 4 and Experiment 7 throughout time points PAS2-Post3 ( $p<0.05$, Fig. $4 D)$.

In summary, Experiments $4-7$ strongly suggest that priming with near-simultaneous bilateral M1 stimulation is necessary for subsequent successful induction of long-term associative plasticity in the SMA-M1 network. 
A

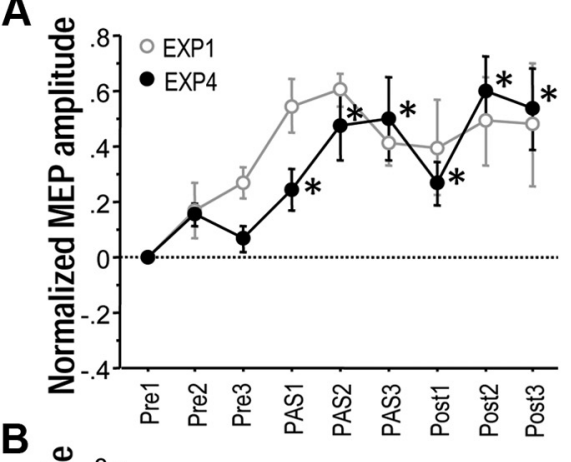

B

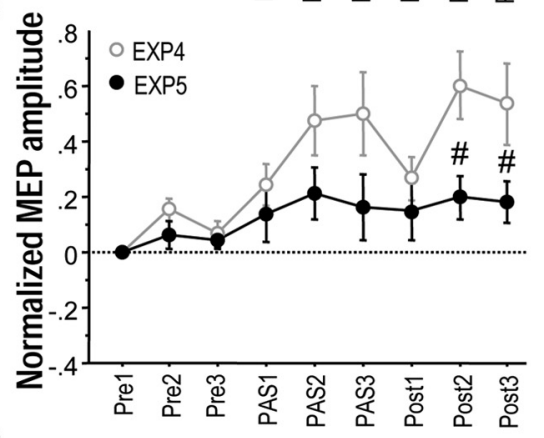

C

D
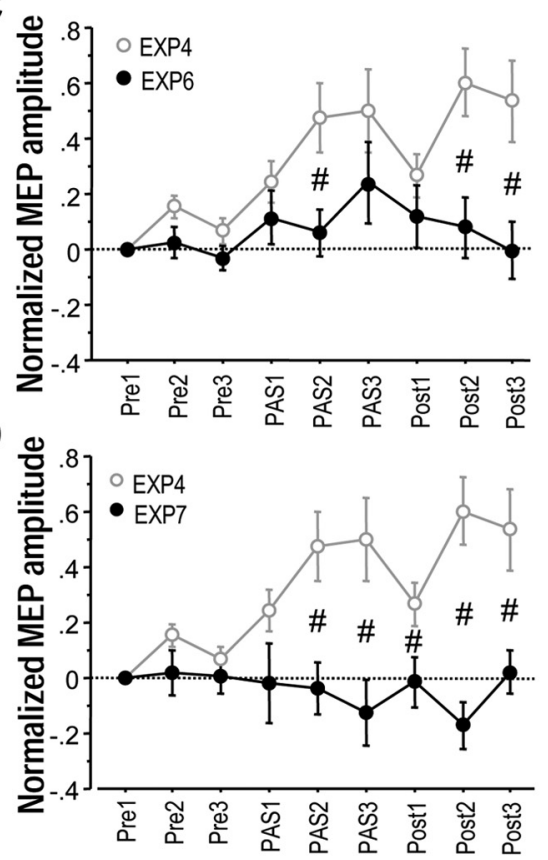

Time points of MEP measurements

Figure 4. Priming by near-synchronous bilateral M1 stimulation is necessary for induction of associative plasticity between SMA and M1 (Experiments 4-7). $A$, MEP changes in left M1 before (Pre1-3), during (PAS1-3) and after (Post1-3) paired associative stimulation of SMA and unilateral left M1 (Experiment 4, black circles). The ISI between SMA and left M1 stimulation in this and all other experiments of this Figure was $-6 \mathrm{~ms}$. During Pre $1-3$ near-simultaneous bilateral M1 stimulation was conducted, during Post1-3 only left M1 was stimulated. Data are compared with paired associative stimulation of SMA with bilateral M1 (Experiment 1, gray circles). Note that there was no difference in MEP change between experimental conditions. In both conditions, MEP amplitude increased significantly during and for at least $30 \mathrm{~min}$ after PAS1-3. Asterisks denote significant MEP changes compared with Pre1 in Experiment $4(p<$ 0.05). B, MEP changes in left M1 before (Pre1-3), during (PAS1-3) and after (Post1-3) paired associative stimulation of SMA and unilateral left M1. During Pre1-3 and Post1-3, only the left M1 was stimulated (Experiment 5, black circles). Here and in $\mathbf{C}$ and $\boldsymbol{D}$, MEP data are compared with those from Experiment 4 (gray circles). Here and in $\boldsymbol{C}$ and $\boldsymbol{D},{ }^{\#} p<0.05$, significant difference in MEP changes at single time points between the experimental conditions. $C$, MEP changes in left M1 before (Pre1-3), during (PAS1-3) and after (Post1-3) associative stimulation of

\section{Discussion}

This study demonstrated for the first time associative plasticity at the systems level of the human SMA-M1 network. Characteristics of this form of plasticity were timing-dependent directionality (i.e., lasting increase vs decrease in M1 excitability, depending on the interval of paired SMA-M1 stimulation), topographical specificity (i.e., stimulation of SMA-proper but not pre-SMA was effective) and state dependence (i.e., associative plasticity in the SMA-M1 network required priming by near-simultaneous M1 stimulation to occur). The following paragraphs will discuss these findings in detail.

\section{Timing-dependent bidirectional associative plasticity in the human SMA-M1 network}

The sign of the change in M1 excitability during and after PAS of the SMA-M1 network depended critically on the ISI between SMA-proper and M1 stimulation. The hand representations of SMA-proper and bilateral M1 are densely reciprocally interconnected in monkeys (Muakkassa and Strick, 1979; Luppino et al., 1993; Geyer et al., 2000; Dum and Strick, 2005) and in humans (Fox et al., 1997; Geyer et al., 2000; Bestmann et al., 2003; Matsumoto et al., 2007). Intracortical microstimulation of SMA-proper in the monkey results in short-latency, likely mono-synaptic excitatory input to $\mathrm{M} 1$ corticospinal neurons peaking at $2 \mathrm{~ms}$ and 4-6 ms (Tokuno and Nambu, 2000). TMS of M1 excites corticospinal neurons largely indirectly through excitatory interneurons, typically with a delay of 3-4.5 ms compared with direct excitation, if the induced current in M1 is directed from posteriorto-anterior (Di Lazzaro et al., 2008). If similar SMA-M1 conduction times apply to humans as in the monkey, then the ISI of $-6 \mathrm{~ms}$ used in Experiment 1 would be sufficient for excitatory synaptic input from SMA-proper to reach corticospinal neurons in M1 a few milliseconds before action potentials are induced in them by suprathreshold M1 stimulation. Although not yet explored systematically in humans, preliminary paired-coil experiments confirmed that conditioning SMA-proper stimulation leads to significant test MEP increase at an ISI of $-6 \mathrm{~ms}$ (N. Arai, M.-K. Lu, Y. Ugawa, U. Ziemann, unpublished data). Furthermore, for the ISI of $+15 \mathrm{~ms}$ (Experiment 1) action potentials in corticospinal neurons elicited by M1 stimulation would occur before the excitatory synaptic input from SMA-proper stimulation has arrived, resulting in a reversed order of these events in corticospinal neurons.

Repetitive pairing of EPSPs followed by action potentials results in long-term potentiation of synaptic efficacy in principal neurons in slice preparations of rat somatosensory cortex while repetitive pairing of actions potentials followed by EPSPs leads to long-term depression (Markram et al., 1997). This STDP plays a fundamental role in governing direction and magnitude of Hebbian synaptic plasticity in many neuronal networks (Bi and Poo, 2001; Dan and Poo, 2004; Caporale and Dan, 2008). The evidence for STDP-like bidirectional changes in human cortex is so far limited to pairing electrical stimulation of fast-conducting pe-

$\leftarrow$

SMA paired with near-simultaneous stimulation of bilateral M1. During Pre1-3 and Post1-3, only the left M1 was stimulated (Experiment 6, black circles). D, MEP changes in left M1 before (Pre1-3), during (PAS1-3) and after (Post1-3) paired associative stimulation of SMA and unilateral left M1. During Pre1-3, nonsimultaneous bilateral M1 stimulation was delivered (ISI = 5 ms), during Post1-3, only the left M1 was stimulated (Experiment 7, black circles). Of note, lasting MEP increase occurred only if paired associative stimulation of the SMA-M1 network was primed by near-simultaneous bilateral M1 stimulation during Pre1-3. 
ripheral nerve afferents with TMS of the contralateral M1 or primary somatosensory cortex (Müller-Dahlhaus et al., 2010). STDP-like changes in corticocortical networks were so far investigated only for the transcallosal connection between M1 in the two hemispheres. While a long-term MEP increase was observed in the conditioned M1 when the ISI between the pairs of conditioning and test M1 stimulation was $8 \mathrm{~ms}$ (Rizzo et al., 2009) or 15 ms (Koganemaru et al., 2009), a long-term MEP decrease was not obtained at other (shorter) ISIs. Therefore, the present data are the first to demonstrate bidirectional associative plasticity in a human corticocortical network fully compatible with STDP.

The inefficacy of intermediate ISI of $-3.2 \mathrm{~ms}$ and $+10 \mathrm{~ms}$ to induce associative plasticity (Fig. 2) underscores the critical role of timing, similar to ineffective intermediate ISI in the conventional peripheral-cortical PAS protocols (Wolters et al., 2003, 2005).

\section{Topographic specificity of PAS-induced plasticity in the SMA-M1 network}

The pre-SMA has no direct anatomical connections with ipsilateral or contralateral M1 in monkeys (Luppino et al., 1993; Geyer et al., 2000; Liu et al., 2002) or humans (Bestmann et al., 2003; Johansen-Berg et al., 2004; Kim et al., 2010). Accordingly, targeting pre-SMA did not result in PAS-induced plasticity when using an otherwise identical stimulation protocol as over SMA-proper (Fig. 3B). Recent paired-coil TMS studies demonstrated effective connectivity between pre-SMA and M1 via corticocortical and cortical-subcortical routes (Mars et al., 2009; Neubert et al., 2010), but these effects were specifically dependent on motor tasks requiring action reprogramming and for this reason are not at variance with the present results obtained at rest.

Preliminary data from a paired-coil study of our group showed that conditioning stimulation of SMA-proper at $90 \%$ of AMT and ISI of -6 ms does not alter test MEP amplitude (N. Arai, M.-K. Lu, Y. Ugawa, U. Ziemann, unpublished data). SMAproper stimulation at $90 \%$ of AMT intensity during PAS failed to induce associative plasticity (Fig. $3 A$ ), indicating that effective excitation of the SMA-proper to M1 projection is a necessary requirement for induction of associative plasticity in the SMA-M1 network.

\section{Priming by near-synchronous bilateral M1 stimulation is necessary for induction of associative plasticity in the SMA-M1 network}

Importantly, successful induction of associative plasticity between SMA-proper and M1 was not confined to SMA-proper and bilateral M1 stimulation, but could also be obtained if SMAproper stimulation was paired with unilateral (left) M1 stimulation (Experiment 4, Fig. 4A). Further experiments established a critical dependence of induction of associative plasticity in the SMA-M1 network on priming with near-simultaneous bilateral M1 stimulation (Experiments 5-7, Fig. 4B-D). This constitutes a significant difference to associative plasticity successfully induced without priming by other peripheral-cortical (Müller-Dahlhaus et al., 2010) or corticocortical (Koganemaru et al., 2009; Rizzo et al., 2009) PAS protocols. In the present experiments, priming never resulted in any overt M1 excitability change as indexed by stable MEP amplitudes over the course of the Pre1-3 blocks. In agreement, one other study showed that repetitive nearsimultaneous bilateral M1 stimulation at an ISI of $1 \mathrm{~ms}$ does not alter MEP amplitude (Rizzo et al., 2009). The statement that priming failed to induce overt M1 excitability change may be qualified by the fact that paired-pulse TMS measures of intracor- tical inhibition and facilitation were not tested. It is, however, unlikely that changes in intracortical inhibition or facilitation have occurred because previous peripheral-cortical (MüllerDahlhaus et al., 2010) and corticocortical (Rizzo et al., 2009) PAS protocols never found such changes, even not in the presence of significant MEP change.

A much more plausible, although at this stage speculative explanation is that the priming induced changes of excitability in SMA-proper which were necessary for induction of associative plasticity in the SMA-M1 network. SMA-proper receives input not only from ipsilateral but also from contralateral M1 (Liu et al., 2002). A subpopulation of SMA neurons may exist which receives input from both ipsi- and contralateral M1, either directly or via the abundant callosal connections between SMAproper of the two hemispheres (Liu et al., 2002). Activation of these neurons by near-simultaneous bilateral M1 stimulation during priming could have resulted in excitability change of these neurons making them more susceptible to subsequent associative stimulation. The critical role of the near-simultaneous bilateral M1 stimulation can be inferred from the findings that priming with unilateral (Fig. $4 B, C$ ) or nonsimultaneous bilateral M1 stimulation (Fig. $4 D$ ) were not effective in resulting in long-term associative plasticity in the SMA-M1 network. One possible test to support this idea of susceptibility change of SMA neurons by near-simultaneous input further would be to demonstrate that simply applying higher SMA-proper stimulation intensity during PAS is not sufficient, in the absence of priming, to result in such long-term associative plasticity.

SMA-proper contains a high proportion of neurons that are specifically active during the preparation and performance of bimanual rather than unimanual movements (Kermadi et al., 1998). Lesions of SMA-proper in monkeys (Brinkman, 1984) and disruptive TMS of the SMA-proper in humans impair particularly the performance of bimanual motor tasks (Obhi et al., 2002; Serrien et al., 2002; Steyvers et al., 2003) and intermanual transfer of procedural knowledge (Perez et al., 2008). On the other hand, preparatory neuronal activity for unimanual movements starts off with bilateral activation of the SMA-proper (Shibasaki and Hallett, 2006). These studies signify the highly convergent and divergent bilateral organization of the SMA-M1 network to enable complex motor behavior. It is of particular interest that SMA-M1 effective connectivity directly determines M1 excitability (Sarfeld et al., 2011) and that normalization of disturbed SMA-M1 network connectivity after cerebral stroke is associated with improvement in motor abilities (Grefkes et al., 2010; Wang et al., 2011). The present findings introduce the possibility of particularly effective bidirectional SMA-M1 network modification by multiple-coil associative stimulation that is governed by the principles of state dependence and timing dependence. Multiple-coil associative stimulation may have therapeutic potential to support purposeful modification of the SMA-M1 network in neurological disorders in which dysfunction of this network contributes to the clinical impairment.

In summary, findings demonstrate for the first time timingdependent bidirectional associative plasticity in the human SMA-M1 network which is in full agreement with spike-timingdependent plasticity. Priming by near-simultaneous M1 stimulation, likely resulting in altered excitability of SMA-proper neurons, is necessary for this associative plasticity to occur, a feature which reflects the dense reciprocal and interhemispheric connectivity of this network. 


\section{References}

Bestmann S, Baudewig J, Siebner HR, Rothwell JC, Frahm J (2003) Subthreshold high-frequency TMS of human primary motor cortex modulates interconnected frontal motor areas as detected by interleaved fMRITMS. Neuroimage 20:1685-1696.

Bi G, Poo M (2001) Synaptic modification by correlated activity: Hebb's postulate revisited. Annu Rev Neurosci 24:139-166.

Brinkman C (1984) Supplementary motor area of the monkey's cerebral cortex: short- and long-term deficits after unilateral ablation and the effects of subsequent callosal section. J Neurosci 4:918-929.

Caporale N, Dan Y (2008) Spike timing-dependent plasticity: a Hebbian learning rule. Annu Rev Neurosci 31:25-46.

Cincotta M, Borgheresi A, Jung P, Balestrieri F, Giovannelli F, Zaccara G, Ziemann U (2005) Physical interactions between induced electrical fields can have substantial effects on neuronal excitation during simultaneous TMS of two brain areas. Clin Neurophysiol 116:1733-1742.

Civardi C, Cantello R, Asselman P, Rothwell JC (2001) Transcranial magnetic stimulation can be used to test connections to primary motor areas from frontal and medial cortex in humans. Neuroimage 14:1444-1453.

Dan Y, Poo MM (2004) Spike timing-dependent plasticity of neural circuits. Neuron 44:23-30.

Di Lazzaro V, Ziemann U, Lemon RN (2008) State of the art: physiology of transcranial motor cortex stimulation. Brain Stimul 1:345-362.

Dum RP, Strick PL (2005) Frontal lobe inputs to the digit representations of the motor areas on the lateral surface of the hemisphere. J Neurosci 25:1375-1386.

Fink GR, Frackowiak RS, Pietrzyk U, Passingham RE (1997) Multiple nonprimary motor areas in the human cortex. J Neurophysiol 77:2164-2174.

Fox P, Ingham R, George MS, Mayberg H, Ingham J, Roby J, Martin C, Jerabek P (1997) Imaging human intra-cerebral connectivity by PET during TMS. Neuroreport 8:2787-2791.

Geyer S, Matelli M, Luppino G, Zilles K (2000) Functional neuroanatomy of the primate isocortical motor system. Anat Embryol (Berl) 202:443-474.

Grefkes C, Nowak DA, Eickhoff SB, Dafotakis M, Küst J, Karbe H, Fink GR (2008) Cortical connectivity after subcortical stroke assessed with functional magnetic resonance imaging. Ann Neurol 63:236-246.

Grefkes C, Nowak DA, Wang LE, Dafotakis M, Eickhoff SB, Fink GR (2010) Modulating cortical connectivity in stroke patients by rTMS assessed with fMRI and dynamic causal modelling. Neuroimage 50:234-243.

Johansen-Berg H, Behrens TE, Robson MD, Drobnjak I, Rushworth MF, Brady JM, Smith SM, Higham DJ, Matthews PM (2004) Changes in connectivity profiles define functionally distinct regions in human medial frontal cortex. Proc Natl Acad Sci U S A 101:13335-13340.

Kermadi I, Liu Y, Tempini A, Calciati E, Rouiller EM (1998) Neuronal activity in the primate supplementary motor area and the primary motor cortex in relation to spatio-temporal bimanual coordination. Somatosens Mot Res 15:287-308.

Kim JH, Lee JM, Jo HJ, Kim SH, Lee JH, Kim ST, Seo SW, Cox RW, Na DL, Kim SI, Saad ZS (2010) Defining functional SMA and pre-SMA subregions in human MFC using resting state fMRI: functional connectivitybased parcellation method. Neuroimage 49:2375-2386.

Koganemaru S, Mima T, Nakatsuka M, Ueki Y, Fukuyama H, Domen K (2009) Human motor associative plasticity induced by paired bihemispheric stimulation. J Physiol 587:4629-4644.

Liu J, Morel A, Wannier T, Rouiller EM (2002) Origins of callosal projections to the supplementary motor area (SMA): a direct comparison between preSMA and SMA-proper in macaque monkeys. J Comp Neurol 443:71-85.

Luppino G, Matelli M, Camarda R, Rizzolatti G (1993) Corticocortical connections of area F3 (SMA-proper) and area F6 (pre-SMA) in the macaque monkey. J Comp Neurol 338:114-140.

Markram H, Lübke J, Frotscher M, Sakmann B (1997) Regulation of synaptic efficacy by coincidence of postsynaptic APs and EPSPs. Science 275:213-215.

Mars RB, Klein MC, Neubert FX, Olivier E, Buch ER, Boorman ED, Rushworth MF (2009) Short-latency influence of medial frontal cortex on primary motor cortex during action selection under conflict. J Neurosci 29:6926-6931.

Matsumoto R, Nair DR, LaPresto E, Bingaman W, Shibasaki H, Lüders HO (2007) Functional connectivity in human cortical motor system: a cortico-cortical evoked potential study. Brain 130:181-197.

Matsunaga K, Maruyama A, Fujiwara T, Nakanishi R, Tsuji S, Rothwell JC (2005) Increased corticospinal excitability after $5 \mathrm{~Hz}$ rTMS over the human supplementary motor area. J Physiol 562:295-306.
Muakkassa KF, Strick PL (1979) Frontal lobe inputs to primate motor cortex: evidence for four somatotopically organized 'premotor' areas. Brain Res 177:176-182.

Müller-Dahlhaus F, Ziemann U, Classen J (2010) Plasticity resembling spike-timing dependent synaptic plasticity: the evidence in human cortex. Front Syn Neurosci 2:1-11.

Nachev P, Kennard C, Husain M (2008) Functional role of the supplementary and pre-supplementary motor areas. Nat Rev Neurosci 9:856-869.

Neubert FX, Mars RB, Buch ER, Olivier E, Rushworth MF (2010) Cortical and subcortical interactions during action reprogramming and their related white matter pathways. Proc Natl Acad Sci U S A 107:13240-13245.

Obhi SS, Haggard P, Taylor J, Pascual-Leone A (2002) rTMS to the supplementary motor area disrupts bimanual coordination. Motor Control 6:319-332.

Oldfield RC (1971) The assessment and analysis of handedness: the Edinburgh inventory. Neuropsychologia 9:97-113.

Perez MA, Tanaka S, Wise SP, Willingham DT, Cohen LG (2008) Timespecific contribution of the supplementary motor area to intermanual transfer of procedural knowledge. J Neurosci 28:9664-9669.

Picard N, Strick PL (1996) Motor areas of the medial wall: a review of their location and functional activation. Cereb Cortex 6:342-353.

Rizzo V, Siebner HS, Morgante F, Mastroeni C, Girlanda P, Quartarone A (2009) Paired associative stimulation of left and right human motor cortex shapes interhemispheric motor inhibition based on a Hebbian mechanism. Cereb Cortex 19:907-915.

Rouiller EM, Babalian A, Kazennikov O, Moret V, Yu XH, Wiesendanger M (1994) Transcallosal connections of the distal forelimb representations of the primary and supplementary motor cortical areas in macaque monkeys. Exp Brain Res 102:227-243.

Sakai K, Ugawa Y, Terao Y, Hanajima R, Furubayashi T, Kanazawa I (1997) Preferential activation of different I waves by transcranial magnetic stimulation with a figure-of-eight shaped coil. Exp Brain Res 113:24-32.

Sarfeld AS, Diekhoff S, Wang LE, Liuzzi G, Uludag K, Eickhoff SB, Fink GR, Grefkes C (2011) Convergence of human brain mapping tools: Neuronavigated TMS Parameters and fMRI activity in the hand motor area. Hum Brain Mapp. Advance online publication. Retrieved April 29, 2011. doi:10.1002/hbm.21272.

Serrien DJ, Strens LH, Oliviero A, Brown P (2002) Repetitive transcranial magnetic stimulation of the supplementary motor area (SMA) degrades bimanual movement control in humans. Neurosci Lett 328:89-92.

Shibasaki H, Hallett M (2006) What is the Bereitschaftspotential? Clin Neurophysiol 117:2341-2356.

Steyvers M, Etoh S, Sauner D, Levin O, Siebner HR, Swinnen SP, Rothwell JC (2003) High-frequency transcranial magnetic stimulation of the supplementary motor area reduces bimanual coupling during anti-phase but not in-phase movements. Exp Brain Res 151:309-317.

Talairach J, Tournoux P (1988) Co-planar stereotaxic atlas of the human brain: 3-dimensional proportional system. An approach to cerebral imaging. New York: Thieme.

Tokuno H, Nambu A (2000) Organization of nonprimary motor cortical inputs on pyramidal and nonpyramidal tract neurons of primary motor cortex: an electrophysiological study in the macaque monkey. Cereb Cortex 10:58-68.

Vorobiev V, Govoni P, Rizzolatti G, Matelli M, Luppino G (1998) Parcellation of human mesial area 6: cytoarchitectonic evidence for three separate areas. Eur J Neurosci 10:2199-2203.

Wang LE, Fink GR, Diekhoff S, Rehme AK, Eickhoff SB, Grefkes C (2011) Noradrenergic enhancement improves motor network connectivity in stroke patients. Ann Neurol 69:375-388.

Wolters A, Sandbrink F, Schlottmann A, Kunesch E, Stefan K, Cohen LG, Benecke R, Classen J (2003) A temporally asymmetric Hebbian rule governing plasticity in the human motor cortex. J Neurophysiol 89:2339-2345.

Wolters A, Schmidt A, Schramm A, Zeller D, Naumann M, Kunesch E, Benecke R, Reiners K, Classen J (2005) Timing-dependent plasticity in human primary somatosensory cortex. J Physiol 565:1039-1052.

Ziemann U, Rothwell JC, Ridding MC (1996) Interaction between intracortical inhibition and facilitation in human motor cortex. J Physiol 496:873-881.

Zilles K, Schlaug G, Geyer S, Luppino G, Matelli M, Qü M, Schleicher A, Schormann T (1996) Anatomy and transmitter receptors of the supplementary motor areas in the human and nonhuman primate brain. Adv Neurol 70:29-43. 